\title{
REFINED WAVE-TRACKING AND NONLINEAR STABILITY OF VISCOUS LAX SHOCKS*
}

\author{
KEVIN ZUMBRUN ${ }^{\dagger}$
}

\begin{abstract}
We make use of recent improvements in the associated linearized theory to give a more accurate (indeed sharp) accounting of the nonlinear motion of a viscous shock wave under the effects of perturbation. This yields a particularly simple proof of $L^{1} \cap L^{\infty} \rightarrow L^{p}$ nonlinear orbital stability for viscous Lax waves satisfying the spectral stability criterion of Zumbrun and Howard: in particular, for weak Lax shocks in the system case and for arbitrary nonsonic shocks in the scalar case. For scalar shocks, we prove also a sharp pointwise convergence result yielding stability for initial data decaying as $(1+|x|)^{-r}, r \geq 1 / 2$, with temporal decay at the same rate.
\end{abstract}

Introduction. Recently, Goodman and Yip, [GY], have announced preliminary findings suggesting the somewhat surprising result of $L^{1} \cap W^{1,1} \rightarrow L^{p}$ orbital stability at rate $t^{-\frac{1}{2}(1-1 / p)}$ for Lax type viscous shock waves satisfying the spectral stability criterion of $[\mathrm{ZH}]$. Previous results for systems have all required localization, i.e. spatial decay, of initial data of at least order $(1+|x|)^{-3 / 2}[\mathrm{SX}, \mathrm{L}, \mathrm{ZH}]$; for scalar equations, the current best result requires localization $(1+|x|)^{-1}$ to achieve the above temporal rate [HZ.1-2].

The approach of [GY] uses the flux transform of [G.2] to essentially "project out" variations in shock location. Together with the linearized decay bounds of $[\mathrm{ZH}]$, this immediately gives the result at the linearized level. The nonlinear analysis is quite nontrivial, however, requiring rather delicate (nonlinear) weighted $L^{1}$ estimates to control terms arising through inversion of the flux transform (indeed, to the best of our knowledge, at the time of our writing this step is not yet complete). Moreover, for systems, the derivative Green's function bounds of $[\mathrm{ZH}]$ are not sufficient to close the iteration proposed in [GY]; specifically, $\left|G_{y}\right|_{L^{1}(x)}=\mathcal{O}(1)$ and not $\sim t^{-1 / 2}$ as needed (see notation below). In fact, we suspect that the desired derivative bounds do not hold for the "integrated" equations arising through the flux transform, except in the scalar case, see Remark 3.3 (they do hold for scalar equations by the bounds in [ZH]). This is a substantial obstacle to the application of the methods of [G.2,GY] to the (nonlinear) system case, at least as originally described in [GY].

On the other hand, we have recently shown for the original, "unintegrated" equations that improved $y$-derivative bounds are possible precisely in the Lax and overcompressive case [Z.1]. (For a heuristic explanation of this somewhat subtle phenomenon, see Discussion, Section 3). At the same time, we gave an (unrelated) refined description of dynamics near the shock layer, making possible a sharpened analysis in the untransformed equations. This suggests the possibility of a direct analysis of $L^{1} \rightarrow L^{p}$

* Received January 20, 2000; revised April 4, 2001.

$\dagger$ Indiana University, Department of Mathematics, Rawles Hall 223, 831 East Third Street, Bloomington, IN 47405-7106, USA (kzumbrun@indiana.edu). Thanks to Jonathan Goodman and Aaron Yip for bringing to our attention their work in [GY], which was the direct stimulation for the present analysis. Thanks also to EvaMarie Elliott for the skillful and efficient typing of this manuscript. This work was supported in part under NSF grant number DMS-9706842. 
stability avoiding the complications introduced by the flux transform at the nonlinear level.

Motivated by these new developments, we here make use of the improved Green's function bounds in [Z.1] to refine the shock-tracking scheme of [ZH,HZ.1-2] a direct approach essentially opposite to that of the flux transform method. We immediately recover optimal linearized decay rates for shocks of all types, including the undercompressive variety (the flux transform method yields linearized results for Lax and overcompressive shocks, nonlinear results for scalar Lax shocks). More important, for Lax shock we obtain a truly simple proof of $L^{1} \cap L^{\infty} \rightarrow L^{p}$ nonlinear orbital stability at the rate $t^{-\frac{1}{2}(1-1 / p)}$ conjectured in [G.2,GY], essentially equivalent to that used by Kawashima [Ka] to study stability of constant solutions. We give also an improved (optimal) pointwise analysis in the scalar case.

2. The Scalar Case. For clarity of exposition, we first carry out our argument completely for the scalar case, which for the purposes of this paper exhibits all features present in the general Lax case. Consider a scalar conservation law

$$
u_{t}+f(u)_{x}=\left(b(u) u_{x}\right)_{x}
$$

$u, f, b \in \mathbb{R}^{1}$, and a (without loss of generality) stationary viscous shock solution

$$
u=\bar{u}(x), \lim _{x \rightarrow \pm \infty} \bar{u}(x)=: u_{ \pm}
$$

satisfying

$(\mathcal{H}) \quad f, b \in C^{2}, \quad b>0, \quad d f\left(u_{-}\right)>0>d f\left(u_{+}\right)$.

These are equivalent to the standard hypotheses $(\mathrm{H} 0)-(\mathrm{H} 4)$ of $[\mathrm{ZH}]$. Note that the third hypothesis yields hyperbolicity of $u_{ \pm}$as rest points of the associated traveling wave ODE, hence

$$
\left|\bar{u}_{x}\right|=\mathcal{O}\left(e^{-n|x|}\right), \quad x>0
$$

Linearizing about $\bar{u}(\cdot)$, we obtain the linearized perturbation equation

$$
v_{t}=L v:=-(a v)_{x}-\left(b v_{x}\right)_{x},
$$

where

$$
b(x):=b(\bar{u}(x)), \quad a(x):=d f(\bar{u}(x))-d b(\bar{u}) \bar{u}_{x} ;
$$

We will denote by $a_{ \pm}:=a( \pm \infty), b_{ \pm}:=b( \pm \infty)$ the limiting values of the coefficients. Define

$$
G(x, t ; y):=e^{L t} \delta_{y}(x)
$$

to be the Green's function associated with operator $\left(\partial_{t}-L\right)$. Then, we have the following bounds, proved in [Z.1]:

Proposition 2.1. Under assumptions $(\mathcal{H})$, we have for $y \leq 0$ the decomposition

$$
G=E+S+R
$$


where

$$
\begin{gathered}
E(x, t ; y):=\left(\frac{\bar{u}_{x}(x)}{u_{+}-u_{-}}\right)\left(\operatorname{errfn}\left(\frac{x-y-a_{-} t}{\sqrt{4 b_{-} t}}\right)\right. \\
\left.-\operatorname{errfn}\left(\frac{x-y+a_{-} t}{\sqrt{4 b_{-} t}}\right)\right), \\
S(x, t ; y):=\frac{e^{\frac{-\left(x-y-a_{-} t\right)^{2}}{4 b_{-}}}}{\sqrt{4 \pi b_{-} t}}\left(\frac{e^{x}}{e^{x}+e^{-x}}\right)
\end{gathered}
$$

and

$$
R=\mathcal{O}\left((1+t)^{-1 / 2} e^{-\eta x^{+}}+e^{-\eta|x|}\right) t^{-1 / 2} e^{-\frac{\left(x-y-a_{-} t\right)^{2}}{M t}}
$$

for some $\eta, M>0$, where $x^{+}$denotes the positive part of $x$. Likewise, we have the derivative bounds

$$
\begin{gathered}
\left|R_{x}\right|=\mathcal{O}\left((1+t)^{-1 / 2} t^{-1 / 2} e^{-\eta x^{+}}+e^{-\eta|x|}\right) t^{-1 / 2} e^{-\frac{(x-y-a-t)^{2}}{M t}}, \\
\left|R_{y}\right|=\mathcal{O}\left((1+t)^{-1 / 2} e^{-\eta x^{+}}+e^{-\eta|x|}\right) t^{-1} e^{\frac{\left(x-y-a_{-}\right)^{2}}{M t}}
\end{gathered}
$$

A symmetric decomposition holds for $y \geq 0$.

Bounds (2.8)-(2.12) refine and somewhat simplify bounds obtained in [H.1-2, ZH] by similar methods.

ExAMPLE. In the case of a Burgers Shock, $\bar{u}(x)=-\tanh \left(\frac{x}{2}\right)$, of the scalar Burgers equation $u_{t}+\left(u^{2} / 2\right)_{x}=u_{x x}$, the linearized equation (2.5) can be solved explicitly by linearized Hopf-Cole transformation, [S,N,Z.5,LZ.1,GSZ], to give an exact formula for the Green's function of:

$$
\begin{aligned}
& G(x, t ; y)= \\
& \quad\left[\left(\frac{e^{-\frac{x}{2}}}{e^{\frac{x}{2}}+e^{-\frac{x}{2}}}\right)(4 \pi t)^{-\frac{1}{2}} e^{-\frac{(x-y-t)^{2}}{4 t}}+\left(\frac{e^{\frac{x}{2}}}{e^{\frac{x}{2}}+e^{-\frac{x}{2}}}\right)(4 \pi t)^{-\frac{1}{2}} e^{-\frac{(x-y+t)^{2}}{4 t}}\right] \\
& \quad+\frac{1}{2} \frac{\partial \bar{u}}{\partial x}\left[\operatorname{errfn}\left(\frac{x-y-t}{\sqrt{4 t}}\right)-\operatorname{errfn}\left(\frac{x-y+t}{\sqrt{4 t}}\right)\right] .
\end{aligned}
$$

Interpretation/Discussion. Due to translation-invariance of (2.1), equation (2.4) possesses the stationary mode $\bar{u}_{x}(\cdot)$, corresponding to instantaneous translation of $\bar{u}(\cdot)$. The dominant term $E$ in $G$ reflects excitation of this mode. Note that its time-asymptotic value is simply the "projection"

$$
\bar{u}_{x}\left\langle\frac{1}{u_{+}-u_{-}}, v_{0}\right\rangle
$$

of the initial data onto the "right eigenspace" $\operatorname{Span}\left\{\bar{u}_{x}\right\}$, by $L^{2}$ inner product against the "left eigenvector" $1 /\left(u_{+}-u_{-}\right)$(for rigorous discussion of associated, nonstandard spectral theory, see $[\mathrm{ZH}])$. However, the effects of an exciting signal are not seen 
instantaneously, but rather propagate according to the joint effects of convection and diffusion as reflected by the multiplying $\operatorname{err} f n$ factor.

A simple heuristic explanation is that translation of the shock wave under perturbation is caused by accumulation of mass at the shock layer; the err fn profile records the amount of mass (up to a time-exponentially decaying tail) that has reached point $x$ by time $t$, of a delta-function signal originating at point $y$ and propagating as a convected heat kernel, hence the shock shift seen at $x$. (This refines a similar discussion in [HZ.3] to include the effects of diffusion).

Linearized stability analysis. Evidently, solutions of the linearized equations do not decay, but converge to the stationary subspace $\operatorname{Span}\left\{\bar{u}_{x}\right\}$. To quantify the rate of convergence, we define, the (linear) instantaneous projection:

$$
\begin{aligned}
\varphi(x, t) & :=\left(\frac{\bar{u}_{x}(x)}{u_{+}-u_{-}}\right) \int_{0}^{+\infty}\left(\left(\operatorname{errfn}\left(\frac{-y-a_{+} t}{\sqrt{4 b_{+} t}}\right)-\operatorname{errfn}\left(\frac{-y+a_{+} t}{\sqrt{4 b_{+} t}}\right)\right) v_{0}(y) d y\right. \\
& +\left(\frac{\bar{u}_{x}(x)}{u_{+}-u_{-}}\right) \int_{0}^{+\infty}\left(\operatorname{errfn}\left(\frac{-y-a_{-} t}{\sqrt{4 b_{-} t}}\right)-\operatorname{errfn}\left(\frac{-y+a_{-} t}{\sqrt{4 b_{-} t}}\right)\right) v_{0}(y) d y \\
& =\int_{-\infty}^{+\infty} \tilde{E}(x, y ; t) v_{0}(y) d y
\end{aligned}
$$

where

$$
\begin{gathered}
\tilde{E}(x, t ; y):=\bar{u}_{x}(x) \tilde{e}(y, t):=\bar{u}_{x}(x) e(0, t ; y) \\
E(x, t ; y)=: \bar{u}_{x}(x) e(x, t ; y)
\end{gathered}
$$

Definition (2.15) refines an analogous definition,

$$
\varphi(x, t):=\left(\frac{\bar{u}_{x}(x)}{u_{+}-u_{-}}\right) \int_{\left|a_{-}\right| t \mid}^{\left|a_{+}\right| t} v_{0}(y) d y,
$$

given in $[\mathrm{ZH}]$. The practical advantages of the new definition are twofold: improved accuracy as $t \rightarrow+\infty$, due to the improved description of $E$ given in Proposition 2.1 (i.e. accounting of diffusive effects), and improved regularity as $t \rightarrow 0$, due to the inclusion of the cancelling exponential tail (i.e. second errfn in each integrand). The importance of the former will be seen immediately, in the improved linear decay rates of Proposition 2.4, below; the importance of the latter will be seen later, in the nonlinear analysis (specifically, in the proof of Lemma 2.5, below). Moreover, there is a conceptual advantage in the formal derivation via (2.16)-(2.17), which both clarifies the method and gives a guide for future extension. The choice of kernel $\tilde{e}(y, t):=e(0, t ; y)$ is easily motivated by the principle that $\tilde{E}-E$ should be minimized.

\section{Denoting}

$$
\widetilde{G}:=G-\widetilde{E},
$$

we have: 
LemMA 2.2. Under assumptions $(\mathcal{H})$, there holds for $y \leq 0$, and some $\eta, M$, $C>0$ :

$$
\begin{aligned}
\widetilde{G} & =\mathcal{O}\left(t^{-\frac{1}{2}} e^{-\frac{\left(x-y-a_{-} t\right)^{2}}{M t}}\left(\frac{e^{-\eta x}}{e^{\eta x}+e^{-\eta x}}\right)\right. \\
& \left.+e^{-\eta t} e^{-\eta x} \operatorname{errfn}\left(\frac{C t-|y|}{\sqrt{M t}}\right)\right)
\end{aligned}
$$

and

$$
\begin{aligned}
\widetilde{G}_{y} & =\mathcal{O}\left(t^{-1} e^{-\frac{\left(x-y-a_{-} t\right)^{2}}{M t}}\left(\frac{e^{-\eta x}}{e^{\eta x}+e^{-\eta x}}\right)\right. \\
& \left.+e^{-\eta t} e^{-\eta x} \operatorname{errfn}\left(\frac{C t-|y|}{\sqrt{M t}}\right)\right),
\end{aligned}
$$

with symmetric bounds for $y \geq 0$. (Note: $E$ and thus $\widetilde{E}$ and $\widetilde{G}$ are defined differently for $y \leq 0$ and $y \geq 0$ ).

Proof. Since terms $S, R$ (resp.Sy, Ry) of (2.7) are clearly absorbable in bounds (2.20)-(2.21), we need only check that $(E-\widetilde{E})$ (resp. $\left.\left(E_{y}-\widetilde{E}_{y}\right)\right)$ so absorb. Using $\left|\bar{u}_{x}\right|=\mathcal{O}\left(e^{-\widetilde{\eta}|x|}\right),(2.3)$, we find for $(x) \geq \sqrt{t}$ that

$$
|E|,|\widetilde{E}|,\left|E_{y}\right|,\left|\widetilde{E}_{y}\right| \leq\left|\bar{u}_{x}\right| \leq C e^{(\widetilde{\eta} / 2)|x|} e^{-(\widetilde{\eta} / 2) t} .
$$

For $x-y-a_{-} t<\left|y-a_{-} t\right|+\sqrt{t}$ on the other hand, we have by the Mean Value Theorem that

$$
\left|\operatorname{errfn}\left(\frac{x-y-a_{-} t}{\sqrt{4 b_{-} t}}\right)-\operatorname{errfn}\left(\frac{-y-a_{-} t}{\sqrt{4 b_{-} t}}\right)\right| \leq C|x| t^{-\frac{1}{2}} e^{-{\frac{\left(x-y-a_{-} t\right)^{2}}{M t}}^{2}}
$$

for $C, M$ sufficiently large. Combining, and noting that $|x| e^{-(\tilde{\eta} / 2)|x|} \leq e^{-(\widetilde{\eta} / 4)|x|}$, we obtain the claimed bound with $\eta:=\widetilde{\eta} / 4$.

Corollary 2.3. Under assumptions $(\mathcal{H})$, there holds

$$
\begin{gathered}
\left|\int_{-\infty}^{+\infty} \tilde{G}(\cdot, t ; y) f(y) d y\right|_{L^{p}} \leq C \min \left\{|f|_{L^{p}}, t^{-\frac{1}{2}(1-1 / p)}|f|_{L^{1}}\right\} \\
\left|\int_{-\infty}^{+\infty} \tilde{G}_{y}(\cdot, t ; y) f(y) d y\right|_{L^{p}} \leq C \min \left\{t^{-1 / 2}|f|_{L^{p}}, t^{-\frac{1}{2}(1-1 / p)-1 / 2}|f|_{L^{1}}\right\}
\end{gathered}
$$

for all $t \geq 0, f \in L^{1} \cap L^{p}$, some $C>0$.

Proof. Bounding the first terms in $(2.20),(2.21)$ by $C t^{-1 / 2} e^{-\frac{\left.x-y-a_{-} t\right)^{2}}{M t}}$ and $C t^{-1} e^{-\frac{\left.x-y-a_{-}\right)^{2}}{M t}}$, respectively, we find by Haussdorf-Young inequality that their contributions satisfy the claimed bounds. The contribution of the second (error) terms 
can be bounded using triangle and Hölder inequalities as

$$
\begin{aligned}
& \left|\int_{-\infty}^{+\infty} e^{-\eta t} e^{-\eta x} \operatorname{errfn}\left(\frac{C t-|y|}{\sqrt{M t}}\right) f(y) d y\right|_{L^{p}(x)} \\
\leq & C e^{-\eta t} \int_{-\infty}^{+\infty} \operatorname{errfn}\left(\frac{C t-|y|}{\sqrt{M t}}\right)|f(y)| d y \\
\leq & \min \left\{e^{-\eta t}\left|\operatorname{errfn}\left(\frac{C t-|y|}{\sqrt{M t}}\right)\right| L_{L^{\infty}}|f|_{L^{1}}, e^{-\eta t}\left|\operatorname{err} f n\left(\frac{C t-|y|}{\sqrt{M t}}\right)\right|_{L^{q}}|f|_{L^{p}}\right\},
\end{aligned}
$$

$1 / p+1 / q=1$, hence are bounded by $C e^{-\eta t / 2} \min \left\{|f|_{L^{1}},|f|_{L^{p}}\right\}$.

Proposition 2.4. Let $(\mathcal{H})$ hold. Then, for initial data $v_{0} \in L^{1}$, we have the linear decay bound

$$
|v(\cdot, t)-\varphi(\cdot, t)|_{L^{p}} \leq t^{-\frac{1}{2}(1-1 / p)}\left|v_{0}\right|_{L^{1}}
$$

and, for data decaying as $(1+|x|)^{-r}$, we have

$$
|v(\cdot, t)-\varphi(\cdot, t)|_{L^{p}} \leq t^{-\frac{1}{2}(1 / \tilde{p}-1 / p)}\left|v_{0}\right|_{L^{\tilde{p}}}
$$

for all $p \geq \tilde{p}>1 / r$.

Proof. Noting that

$$
v(x, t)-\varphi(x, t)=\int_{-\infty}^{\infty} \widetilde{G}(x, t, y) v_{0}(y) d y
$$

we immediately obtain claim (2.26) from Corollary 2.3, (2.24). For data decaying as $\left|v_{0}(x)\right| \leq C(1+|x|)^{-r}$, we observe that the same argument yields

$$
\begin{aligned}
\left|\int_{-\infty}^{+\infty} \tilde{G}(\cdot, t ; y) f(y) d y\right|_{L^{p}} & \leq C t^{-\frac{1}{2}(1-1 / s)}|f|_{L^{\tilde{p}}} \\
& \leq C t^{-\frac{1}{2}(1 / \tilde{p}-1 / p)}|f|_{L^{\tilde{p}}}
\end{aligned}
$$

where $1 / s=1+1 / p-1 / \tilde{p}$, thus giving result $(2.27)$.

Proposition 2.4 (which applies for systems also, see next section) sharpens the rates of orbital stability given in Proposition 9.2 of $[\mathrm{ZH}]$, for initial data decaying more slowly than $(1+|x|)^{-r}, r>1$.

Nonlinear Stability Analysis. We now carry out the nonlinear stability argument following the framework set up in [ZH, HZ.1-2]. Define the nonlinear perturbation

$$
v(x, t):=u(x+\delta(t))-\bar{u}(x),
$$

where $\delta(t)$ (estimating shock location) is to be determined later; for definiteness, fix $\delta(0)=0$. Then,

$$
v_{t}-L v=Q\left(v, v_{x}\right)_{x}+\dot{\delta}(t)\left(\bar{u}_{x}+v_{x}\right)
$$

where

$$
Q\left(v, v_{x}\right)=\mathcal{O}\left(|v|^{2}+|v|\left|v_{x}\right|\right)
$$


so long as $|v|$ remains bounded. By Duhamel's principle, and the fact that

$$
\int_{-\infty}^{\infty} G(x, t ; y) \bar{u}_{x}(y) d y=e^{L t} \bar{u}_{x}(x)=\bar{u}_{x}(x)
$$

we have

$$
\begin{aligned}
& v(x, t)=\int_{-\infty}^{\infty} G(x, t ; y) v_{0}(y) d y \\
& -\int_{0}^{t} \int_{-\infty}^{\infty} G_{y}(x, t-s ; y)\left(Q\left(v, v_{x}\right)+\dot{\delta} v\right)(y, s) d y d \\
& +\delta(t) \bar{u}_{x} .
\end{aligned}
$$

Defining, by analogy with the linear case, the nonlinear instantaneous projection:

$$
\begin{aligned}
\varphi(x, t) & :=-\delta(t) \bar{u}_{x} \\
& :=\int_{-\infty}^{\infty} \tilde{E}(x, t ; y) v_{0}(y) d y \\
& -\int_{0}^{t} \int_{-\infty}^{\infty} \widetilde{E}_{y}(x, t-s ; y)\left(Q\left(v, v_{x}\right)+\dot{\delta} v\right)(y, s) d y
\end{aligned}
$$

or equivalently, the instantaneous shock location:

$$
\begin{aligned}
\delta(t) & =-\int_{-\infty}^{\infty} \widetilde{e}(y, t) v_{0}(y) d y \\
& +\int_{0}^{t} \int_{-\infty}^{+\infty} \widetilde{e}_{y}(y, t-s)\left(Q\left(v, v_{x}\right)+\dot{\delta} v\right)(y, s) d y d s
\end{aligned}
$$

where $\tilde{E}, \tilde{e}$ are defined as in (2.16), and recalling (2.19) and (2.8), we thus obtain the reduced equations:

$$
\begin{aligned}
v(x, t) & =\int_{-\infty}^{\infty} \widetilde{G}(x, t ; y) v_{0}(y) \\
& -\int_{0}^{t} \int_{-\infty}^{\infty} \widetilde{G}_{y}(x, t-s ; y)\left(Q\left(v, v_{x}\right)+\dot{\delta} v\right)(y, s) d y
\end{aligned}
$$

and, differentiating (2.35) with respect to $t$,

$$
\begin{aligned}
\dot{\delta}(t) & =-\int_{-\infty}^{\infty} \widetilde{e}_{t}(y, t) v_{0}(y) d y \\
& +\int_{0}^{t} \int_{-\infty}^{+\infty} \widetilde{e}_{y t}(y, t-s)\left(Q\left(v, v_{x}\right)+\dot{\delta} v\right)(y, s) d y d s
\end{aligned}
$$

Note: In deriving (2.37), we have used the fact that $\tilde{e}_{y}(y, s) \rightarrow 0$ as $s \rightarrow 0$, as the difference of approaching heat kernels, in evaluating the boundary term

$$
\int_{-\infty}^{+\infty} \tilde{e}_{y}(y, 0)\left(Q\left(v, v_{x}\right)+\dot{\delta} v\right)(y, t) d y=0 .
$$

(Indeed, $\left|e_{y}(\cdot, s)\right|_{L^{1}} \rightarrow 0$, see Remark 2.6, below). 
The defining relation $\delta(t) \bar{u}_{x}:=-\varphi$ in (2.34) can be motivated heuristically by

$$
\begin{aligned}
\bar{v}(x, t)-\varphi(x, t) \sim v & =u(x+\delta(t), t)-\bar{u}(x) \\
& \sim \bar{v}(x, t)+\delta(t) \bar{u}_{x}(x),
\end{aligned}
$$

where $\bar{v}$ denotes the solution of the linearized perturbation equations. Alternatively, it can be thought of as the requirement that the instantaneous projection of the shifted (nonlinear) perturbation variable $v$ be zero, [HZ.1-2].

REMARK. Comparing (2.34) to (2.15), we see that the first term in (2.35) corresponds to linear movement of the shock, dependent only on the initial data. The second term, on the other hand, corresponds to nonlinear movement, and evolves dynamically. This represents a new level of detail in the tracking of viscous shock waves. Previous analyses (e.g. in [LZ.2,HZ.2-3]) were not sufficiently fine to capture the nonlinear movement of Lax-type (e.g. diffusive scalar) shock waves.

LEMMA 2.5. The kernel ẽ satisfies

$$
\begin{gathered}
\left|\tilde{e}_{y}(\cdot, t)\right|_{L^{p}},\left|\tilde{e}_{t}(\cdot, t)\right|_{L^{p}} \leq C t^{-\frac{1}{2}(1-1 / p)}, \\
\left|\tilde{e}_{t y}(\cdot, t)\right|_{L^{p}} \leq C t^{-\frac{1}{2}(1-1 / p)-1 / 2},
\end{gathered}
$$

for all $t>0$. Moreover, for $y \leq 0$ we have the pointwise bounds

$$
\begin{gathered}
\left|\tilde{e}_{y}(y, t)\right|,\left|\tilde{e}_{t}(y, t)\right| \leq C t^{-\frac{1}{2}} e^{-\frac{\left(y+a_{-} t\right)^{2}}{M t}}, \\
\left|\tilde{e}_{t y}(y, t)\right| \leq C t^{-1} e^{-\frac{\left(y+a_{-} t\right)^{2}}{M t}},
\end{gathered}
$$

for $M>0$ sufficiently large (i.e. $>4 b_{ \pm}$), and symmetrically for $y \geq 0$.

Proof. From (2.8), (2.16), we have for $y \leq 0$ the explicit representation

$$
\tilde{e}(y, t)=\left(\frac{1}{u_{+}-u_{-}}\right)\left(\operatorname{errfn}\left(\frac{-y-a_{-} t}{\sqrt{4 b_{-} t}}\right)-\operatorname{errfn}\left(\frac{-y+a_{-} t}{\sqrt{4 b_{-} t}}\right)\right),
$$

and symmetrically for $y \geq 0$. Thus,

$$
\begin{gathered}
\tilde{e}_{y}(y, t)=\left(\frac{1}{u_{+}-u_{-}}\right)\left(K\left(y+a_{-} t, t\right)-K\left(y-a_{-} t, t\right)\right) \\
\tilde{e}_{t}(y, t)=\left(\frac{1}{u_{+}-u_{-}}\right)\left(\left(K+K_{y}\right)\left(y+a_{-} t, t\right)-\left(K+K_{y}\right)\left(y-a_{-} t, t\right)\right), \\
\tilde{e}_{t y}(y, t)=\left(\frac{1}{u_{+}-u_{-}}\right)\left(\left(K_{y}+K_{y y}\right)\left(y+a_{-} t, t\right)-\left(K_{y}+K_{y y}\right)\left(y-a_{-} t, t\right)\right),
\end{gathered}
$$

where

$$
K(y, t):=\frac{e^{-y^{2} / 4 b_{-} t}}{\sqrt{4 \pi b_{-} t}}
$$


denotes an appropriate heat kernel. The pointwise bounds (2.41)-(2.42) follow immediately for $t \geq 1$ by properties of the heat kernel, in turn yielding (2.39)-(2.40) in this case. The bounds for small time $t \leq 1$ follow from estimates

$$
\begin{aligned}
\left|K_{y}\left(y+a_{-} t, t\right)-K_{y}\left(y-a_{-} t, t\right)\right| & =\left|\int_{y+a_{-} t}^{y-a_{-} t} K_{y y}(z, t) d z\right| \\
& \leq C t^{-3 / 2} \int_{y+a_{-} t}^{y-a_{-} t} e^{\frac{-z^{2}}{M t}} d z \\
& \leq C t^{-1 / 2} e^{-\frac{\left(y+a_{-} t\right)^{2}}{M t}}
\end{aligned}
$$

and, similarly,

$$
\begin{aligned}
\left|K_{y y}\left(y+a_{-} t, t\right)-K_{y y}\left(y-a_{-}, t\right)\right| & =\left|\int_{-a_{-} t}^{a_{-} t} K_{y y y}(z, t) d z\right| \\
& \leq C t^{-2} \int_{y+a_{-} t}^{y-a_{-} t} e^{\frac{-z^{2}}{M t}} d z \\
& \leq C t^{-1} e^{-\frac{\left(y+a_{-} t\right)^{2}}{M t}} .
\end{aligned}
$$

The bounds for $\left|\tilde{e}_{y}\right|$ are again immediate. Note that we have taken crucial account of cancellation in the small time estimates of $\tilde{e}_{t}, \tilde{e}_{t y}$.

REMARK 2.6. For $t \leq 1$, a calculation analogous to that of (2.48) yields $\left|\tilde{e}_{y}(y, t)\right| \leq$ $C e^{-\frac{\left(y+a_{-} t\right)^{2}}{M t}}$, and thus $|e(\cdot, s)|_{L^{1}} \rightarrow 0$ as $s \rightarrow 0$.

With these preparations, we easily obtain our main result:

Theorem 2.7. Let $(\mathcal{H})$ hold, and $\left|v_{0}\right|_{L^{1}},\left|v_{0}\right|_{L^{\infty}} \leq \zeta_{0}$, $\zeta_{0}$ sufficiently small. Then, the solutions $(v, \delta)(x, t)$ of (2.30), (2.35) with initial data $v_{0}$ satisfy:

$$
\begin{gathered}
|v(\cdot, t)|_{L^{p}} \leq C \zeta_{0}(1+t)^{-\frac{1}{2}(1-1 / p)}, \\
|\dot{\delta}(t)| \leq C \zeta_{0}(1+t)^{-1 / 2}, \\
|\delta(t)| \leq C \zeta_{0} .
\end{gathered}
$$

Proof. Defining

$$
\zeta(t):=\sup _{0 \leq s \leq t, p}|v(\cdot, s)|_{L^{p}}(1+s)^{\frac{1}{2}(1-1 / p)}+\sup _{0 \leq s \leq t}|\delta(s)|(1+s)^{\frac{1}{2}}
$$

we obtain from (2.24)-(2.25)

$$
\begin{aligned}
|v(\cdot, t)|_{L^{p}} & \leq C t^{-\frac{1}{2}(1-1 / p)}\left|v_{0}\right|_{L^{1}} \\
& \left.+\int_{0}^{t / 2}(t-s)^{-\frac{1}{2}(1-1 / p)-\frac{1}{2}} \mid Q+\dot{\delta} v\right)\left.\right|_{L^{1}}(s) d s \\
& +\int_{t / 2}^{t}(t-s)^{-\frac{1}{2}}|(Q+\dot{\delta} v)|_{L^{p}}(s) d s .
\end{aligned}
$$



that

By standard short-time estimates, we have, so long as $|v|_{L^{\infty}}$ remains bounded,

$$
\left|v_{x}(\cdot, t)\right|_{L^{\infty}} \leq \begin{cases}C|v(\cdot, t-1)|_{L^{\infty}}, & \text { for } t \geq 1 \\ C t^{-\frac{1}{2}}\left|v_{0}\right|_{L^{\infty}}, & \text { for } t \leq 1 .\end{cases}
$$

Thus, recalling (2.31), (2.53), we can bound

$$
\begin{aligned}
\left|\left(Q\left(v, v_{x}\right)+\dot{\delta} v\right)(\cdot, t)\right|_{L^{p}} & \leq\left(\left|v_{x}\right|_{L^{\infty}}+|v|_{L^{\infty}}+|\dot{\delta}|\right)|v|_{L^{p}} \\
& \leq C \zeta^{2} t^{-\frac{1}{2}}(1+t)^{-\frac{1}{2}(1-1 / p)}
\end{aligned}
$$

Substituting into (2.54), we obtain for $t \geq 1$ :

$$
\begin{aligned}
|v(\cdot, t)|_{L^{p}} & \leq C \zeta_{0} t^{-\frac{1}{2}(1-1 / p)} \\
& +C \zeta(t)^{2}\left(\int_{0}^{t / 2}(t-s)^{-\frac{1}{2}(1-1 / p)-\frac{1}{2}} s^{-\frac{1}{2}} d s\right. \\
& \left.+\int_{t / 2}^{t}(t-s)^{-\frac{1}{2}}(1+s)^{-\frac{1}{2}(1-1 / p)} s^{-\frac{1}{2}}\right) \\
& \leq C\left(\zeta_{0}+\zeta(t)^{2}\right) t^{-\frac{1}{2}(1-1 / p)}
\end{aligned}
$$

For $t \leq 1$, we can use instead the bound

$$
\begin{aligned}
|v(\cdot, t)|_{L^{p}} & \leq C\left|v_{0}\right|_{L^{p}}+\int_{0}^{t}(t-s)^{-\frac{1}{2}}|Q+\dot{\delta} v|_{L^{p}}(s) d s \\
& \leq C \zeta_{0}+\zeta(t)^{2} \int_{0}^{t}(t-s)^{-\frac{1}{2}}(1+s)^{-\frac{1}{2}(1-1 / p)-\frac{1}{2}} d s \\
& \leq C\left(\zeta_{0}+\zeta(t)^{2}\right)
\end{aligned}
$$

Combining and rearranging, we have

$$
|v(\cdot, t)|_{L^{p}}(1+t)^{\frac{1}{2}(1-1 / p)} \leq C\left(\zeta_{0}+\zeta(t)^{2}\right)
$$

In precisely the same fashion, we obtain from (2.37) and (2.39)-(2.40) the bound

$$
|\delta(t)|(1+t)^{1 / 2} \leq C\left(\zeta_{0}+\zeta(t)^{2}\right),
$$

giving

$$
\zeta(t) \leq C\left(\zeta_{0}+\zeta(t)^{2}\right) .
$$

But, this yields $\zeta(t) \leq 2 C \zeta_{0}$ for all $t \geq 0$, for $\zeta_{0}$ sufficiently small, by continuous indication. Comparing with definition (2.53), we obtain the results (2.50)-(2.51).

Similarly, we obtain (2.52) from representation (2.35) together with (2.45), (2.39), the evident bound $|\widetilde{e}(\cdot, t)| \leq C,\left|v_{0}\right|_{L^{1}} \leq \zeta_{0}$, and the already established bound

$$
|Q+\dot{\delta} v|_{L^{1}}(t) \leq C \zeta_{0}^{2} t^{-\frac{1}{2}}
$$

Corollary 2.8. Let $(\mathcal{H})$ hold. Then, for $\left|u_{0}-\bar{u}\right|_{L^{1}},\left|u_{0}-\bar{u}\right|_{L^{\infty}} \leq \zeta_{0}$ sufficiently small, the solution $u(x, t)$ of (2.1) with initial data $u_{0}$ satisfies

$$
|u(x, t)-\bar{u}(x-\delta(t))|_{L^{p}} \leq C \zeta_{0}(1+t)^{-\frac{1}{2}(1-1 / p)}
$$


for $\delta(t)$ satisfying

$$
\begin{gathered}
|\dot{\delta}(t)| \leq C \zeta_{0}(1+t)^{-1 / 2}, \\
|\delta(t)| \leq C \zeta_{0} .
\end{gathered}
$$

Remarks. 1. The bound (2.64) follows also from $|u(x, t)-\bar{u}(x-\delta(t))|_{L^{1}} \leq C \zeta_{0}$ and conservation of mass.

2. For $b(u) \equiv$ constant, we can remove the assumption $\left|v_{0}\right|_{L^{\infty}} \leq \zeta_{0}$, taking $\left|v_{0}\right|_{L^{\infty}}$ merely bounded, to obtain the degraded bounds $C \zeta_{0} t^{-\frac{1}{2}(1-1 / p)}$ in place of $C \zeta_{0}(1+t)^{-\frac{1}{2}(1-1 / p)}$.

3. Bound (2.62) is a sharp rate for orbital stability, as can be seen by examination of the linearized stability analysis. Likewise, (2.64) gives the sharp result that convergence to the time-asymptotic state predicted by conservation of mass may be arbitrarily slow. Such convergence eventually occurs for scalar equations, by $L^{1}$ contraction [FS]; however, for systems, it is an interesting question whether $\delta(t)$ approaches a limit, or not. (Note: the bound (2.63) yields still poorer information, allowing oscillations of up to $\left.\mathcal{O}\left(t^{\frac{1}{2}}\right) \rightarrow \infty\right)$.

Pointwise bounds. Using the pointwise bounds of Lemma 2.2 and Lemma 2.5, we can easily obtain a sharp nonlinear orbital stability result also for non-integrable, but weakly localized data, decaying as $(1+|x|)^{-\frac{1}{2}}$. This result extends and simplifies similar results in [H.1-3, HZ.1-2].

A straightforward computation [H.3, HZ.1-2] yields

LEMMA 2.9. Let $d(x):=(1+|x|)^{-r}, r>0$. Then, for $y \leq 0$,

$$
\begin{aligned}
\int_{-\infty}^{\infty}\left(\frac{e^{-\eta x}}{e^{\eta x}+e^{-\eta x}}\right) t^{-\frac{1}{2}} e^{-\frac{\left(x-y-a_{-}\right)}{M t}} d(y) d y & \\
& \leq C d(|x|+t),
\end{aligned}
$$

(Note: (2.66) is a special case of (2.65), with $x=0$ ).

Theorem 2.10. Assuming $(\mathcal{H})$, let $d(x):=(1+|x|)^{-r}, r \geq 1 / 2$. Then, for $u_{0}$ Hölder continuous, exponent $\widetilde{\alpha}>0$, with $\tilde{\alpha}$-Hölder norm bounded by some prescribed constant, and $\left|u_{0}-\bar{u}\right|(x) \leq \zeta_{0} d(x)$, $\zeta_{0}$ sufficiently small, the solution $u$ of (2.1), with initial data $u_{0}$, satisfies

$$
\begin{gathered}
\left|u(x, t)-\bar{u}_{x}(x-\delta(t))\right| \leq C \zeta_{0} d(|x|+t), \\
|\dot{\delta}(t)| \leq C \zeta_{0} d(t),
\end{gathered}
$$

for some $C\left(\zeta_{0}\right)>0$ independent of $u_{0}$. 
Proof. Proceeding similarly as before, introduce the nonlinear perturbation variable $v$ and shock location $\delta$, satisfying (2.36)-(2.37), and define

$$
\zeta(t):=\sup _{y, 0 \leq s \leq t}|v(y, x)| / d(|y|+s)+\sup _{0 \leq s \leq t}|\delta(s)| / d(s) .
$$

Applying (2.69), bounds (2.20)-(2.21) and (2.41)-(2.42) on $\widetilde{G}$ and $\widetilde{E}$, and the convolution bounds (2.65)-(2.66), we obtain from representations (2.36) and (2.37) the respective bounds

$$
\begin{aligned}
|v(x, t)| & \leq C \zeta_{0} d(|x|+t)+C \zeta(t)^{2} \int_{0}^{t}(t-s)^{-\frac{1}{2}} d(|x|+t) d(s)\left(1+s^{-1 / 2}\right) d s \\
& \leq \widetilde{C}\left(\zeta_{0}+\zeta(t)^{2}\right) d(|x|+t),
\end{aligned}
$$

and

$$
\begin{aligned}
|\dot{\delta}(t)| & \leq C \zeta_{0} d(t)+C \zeta(t)^{2} \int_{0}^{t}(t-s)^{-\frac{1}{2}} d(t) d(s)\left(1+s^{-1 / 2}\right) d s \\
& \leq \widetilde{C}\left(\zeta_{0}+\zeta(t)^{2}\right) d(t)
\end{aligned}
$$

where in both cases we have used $|d(s)|=\mathcal{O}\left((1+s)^{-1 / 2}\right)$ to obtain $\int_{0}^{t}(t-s)^{-1 / 2} d(s)(1+$ $\left.s^{-1 / 2}\right) d s \leq C$. Here, in place of (2.55), we have used the pointwise short time theory of $[\mathrm{ZH}]$, section 11 , to bound

$$
\begin{aligned}
\left|v_{x}(x, t)\right| & \leq C \begin{cases}\zeta(t-1) d(|x|+t-1), & t \geq 1 \\
\zeta_{0} t^{-\frac{1}{2}} d(x), & t \leq 1\end{cases} \\
& \leq C \zeta(t) d(|x|+t)\left(1+t^{-1 / 2}\right)
\end{aligned}
$$

As in the previous argument, this yields $\zeta(t) \leq C\left(\zeta_{0}+\zeta(t)^{2}\right)$, and $\zeta(t) \leq 2 \zeta_{0}$ for $\zeta_{0}$ sufficiently small.

Remarks. 1. Theorem 2.10 shows that algebraic spatial decay translates directly into temporal (orbital) decay at the same rate, generalizing the corresponding observation made for exponentially decaying data by Il'in and Olenik [IO].

2. Since mass is unbounded, there is no well-defined time-asymptotic state for data decaying as $(1+|x|)^{-1}$ or slower. Thus, orbital stability is the only relevant notion here. On the other hand, the nonlinear part of $\delta(t)$,

$$
\begin{aligned}
& \int_{0}^{t} \int_{-\infty}^{+\infty} \widetilde{e}_{y}(y, t-s)\left(Q\left(v, v_{x}\right)+\dot{\delta} v\right)(y, s) d y d s \\
\leq & C \zeta(t)^{2} \int_{0}^{t}(t-s)^{-1 / 2} d(s)\left(1+s^{-1 / 2}\right) d s \\
\leq & \tilde{C} \zeta_{0}^{2}
\end{aligned}
$$

is bounded, hence the main contribution to the time-asymptotic location of the shock is the "mass distribution function" given by linear estimate (2.15), which can vary as much as $t d(t) \sim t^{1 / 2}$ as $t \rightarrow \infty$.

3. Hölder continuity is used only in the short-time theory leading to [GS.1], based on the parametrix method of Levi [Fr,Le], see discussion [ZH]. It can be dropped for 
$b \equiv$ constant.

3. The System Case. Now, consider the general situation of a stationary viscous shock solution

$$
u=\bar{u}(x), \quad \lim _{x \rightarrow \pm} \bar{u}(x)=: u_{ \pm}
$$

of a system of viscous conservation laws

$$
u_{+}+f(u)_{x}=\left(B(u) u_{x}\right)_{x}
$$

$u, f \in \mathbb{R}^{n}, B \in \mathbb{R}^{n \times n}$, i.e. a solution of the traveling wave ODE

$$
\bar{u}^{\prime}=B(\bar{u})^{-1}\left(f(\bar{u})-f\left(u_{-}\right)\right) .
$$

Following $[\mathrm{ZH}]$, we make assumptions $(\mathcal{H})$ below, generalizing those of the scalar case:

(H0) $f, B \in C^{2}$.

(H1) $\quad \operatorname{Re} \sigma(B)>0$.

(H2) $\quad \sigma\left(f^{\prime}\left(u_{ \pm}\right)\right)$real, distinct, and nonzero.

(H3) $\operatorname{Re} \sigma\left(-i k f^{\prime}\left(u_{ \pm}\right)-k^{2} B\left(u_{ \pm}\right)\right)<-\theta k^{2}$ for all real $k$, some $\theta>0$.

(H4') The unstable manifold of $u_{-}$in (3.3) is transverse to the stable manifold of $u_{+}$, with one-dimensional intersection $\{\bar{u}(x)\}$. (In particular, the solution $\bar{u}(\cdot)$ of (3.1) $-(3.2)$ is unique up to translation).

Note that (H3)-(H4') are specific to systems, being in the scalar case consequences of (H1)-(H2). Condition (H3) is the stable viscosity matrix criterion of Majda and Pego, corresponding to linearized stability of the constant solutions $u \equiv u_{ \pm}[\mathrm{MP}, \mathrm{K}]$ (clearly necessary for stability of $\bar{u}(\cdot)$ of the type we seek, see further discussion $([\mathrm{ZH}]$, pp. 746, 767, and 774-775). Condition (H4'), specializing ( $\mathrm{H} 4)$ of [ZH], is the requirement that viscous profile $\bar{u}(\cdot)$ be of nondegenerate Lax type (see classification, Section 10.1 of $[\mathrm{ZH}])$; In particular, it implies the Lax characteristic condition [Lax]:

$$
a_{p}^{-}>0>a_{p}^{+} ; \quad \operatorname{sgn} a_{j}^{-}=\operatorname{sgn} a_{j}^{+} \neq 0, j \neq p,
$$

where $p$ is the principal characteristic family of the shock and

$$
a_{1}^{ \pm}<\cdots<a_{n}^{ \pm}
$$

denote the (ordered) eigenvalues of $d f\left(u_{ \pm}\right)$.

Linearizing about $\bar{u}(\cdot)$ gives, similarly as in the scalar case:

$$
v_{t}=L v:=-(A v)_{x}-\left(B v_{x}\right)_{x},
$$

with

$$
B(x):=B\left(\bar{u}_{x}\right), \quad A(x) v:=d f(\bar{u}(x)) v-d B(\bar{u}(x)) v \bar{u}_{x}
$$


Denoting $A_{ \pm}:=A( \pm \infty), B_{ \pm}:=B( \pm \infty)$, define the (scalar) characteristic speeds $a_{1}^{ \pm}<\cdots<a_{n}^{ \pm}$(as above) to be the eigenvalues of $A_{ \pm}$, and the left and right (scalar) characteristic modes $l_{j}^{ \pm}, r_{j}^{ \pm}$to be corresponding left and right eigenvectors, respectively, normalized so that $l_{j} \cdot r_{k}=\delta_{k}^{j}$. Following Kawashima [K], define associated effective scalar diffusion rates $\beta_{j}^{ \pm}: j=1, \cdots, n$ by relation

$$
\left(\begin{array}{ccc}
\beta_{1}^{ \pm} & & 0 \\
& \vdots & \\
0 & & \beta_{n}^{ \pm}
\end{array}\right)=\operatorname{diag} L_{ \pm} B_{ \pm} R_{ \pm}
$$

where $L_{ \pm}:=\left(l_{1}^{ \pm}, \ldots, l_{n}^{ \pm}\right)^{t}, R_{ \pm}:=\left(r_{1}^{ \pm}, \ldots, r_{n}^{ \pm}\right)$diagonalize $A_{ \pm}$.

As previously, define

$$
G(x, t ; y):=e^{L t} \delta_{y}(x)
$$

to be the Green's function associated with $\left(\partial_{t}-L\right)$. Then, the relevant linearized theory can be summarized in the following two propositions, proved in $[\mathrm{ZH}],[\mathrm{Z} .1]$, respectively:

Proposition 3.1. Given $(\mathcal{H})$, necessary conditions for $L^{p}$-linearized orbital stability, $p>0$, of $\bar{u}(\cdot)$ with respect to perturbations $v_{0} \in C_{0}^{\infty}$ are: $(\mathcal{D})$ :

(D1) L has no ( $L^{2}$, without loss of generality) eigenvalues in $\{R e \lambda \geq 0\} \backslash\{0\}$.

(D2) $\Delta:=\operatorname{det}\left(r_{1}^{-}, \ldots, r_{p-1}^{-}, r_{p+1}^{+}, \ldots, r_{n}^{+}, u_{+}-u_{-}\right) \neq 0$, where $p$ is the principal characteristic field defined in (3.4)-(3.5).

Proposition 3.2. Under assumptions $(\mathcal{H}),(\mathcal{D})$, we have for $y \leq 0$ the decomposition

$$
G=E+S+R
$$

where

$$
\begin{aligned}
E(x, t ; y) & :=\sum_{a_{k}^{-}>0}\left[c_{k,-}^{0}\right] \bar{u}_{x}(x) l_{k}^{-t}\left(\operatorname{err} n\left(\frac{x-y-a_{k}^{-} t}{\sqrt{4 \beta_{k}^{-} t}}\right)\right. \\
& \left.-\operatorname{errfn}\left(\frac{x-y+a_{k}^{-} t}{\sqrt{4 \beta_{k}^{-} t}}\right)\right),
\end{aligned}
$$




$$
\begin{aligned}
S(x, t ; y) & :=\sum_{a_{k}^{-}<0} r_{k}^{-} l_{k}^{-t}\left(4 \pi \beta_{k}^{-} t\right)^{-1 / 2} e^{-\left(x-y-a_{k}^{-} t\right)^{2} / 4 \beta_{k}^{-} t} \\
& +\sum_{a_{k}^{-}>0} r_{k}^{-} l_{k}^{-t}\left(4 \pi \beta_{k}^{-} t\right)^{-1 / 2} e^{-\left(x-y-a_{k}^{-} t\right)^{2} / 4 \beta_{k}^{-} t}\left(\frac{e^{x}}{e^{x}+e^{-x}}\right) \\
& +\sum_{a_{k}^{-}>0, a_{j}^{-}<0} \chi_{\{t \geq 1\}}\left[c_{k,-}^{j,-}\right] r_{j}^{-} l_{k}^{-t}\left(4 \pi \bar{\beta}_{j k}^{-} t\right)^{-1 / 2} e^{-\left(x-z_{j k}^{-}\right)^{2} / 4 \bar{\beta}_{j k}^{-} t}\left(\frac{e^{-x}}{e^{x}+e^{-x}}\right), \\
& +\sum_{a_{k}^{-}>0, a_{j}^{+}>0} \chi_{\{t \geq 1\}}\left[c_{k,-}^{j,+}\right] r_{j}^{+} l_{k}^{-t}\left(4 \pi \bar{\beta}_{j k}^{+} t\right)^{-1 / 2} e^{-\left(x-z_{j k}^{+}\right)^{2} / 4 \bar{\beta}_{j k}^{+} t}\left(\frac{e^{x}}{e^{x}+e^{-x}}\right),
\end{aligned}
$$

with

$$
z_{j k}^{ \pm}(y, t):=a_{j}^{ \pm}\left(t-\frac{|y|}{\left|a_{k}^{-}\right|}\right)
$$

and

$$
\bar{\beta}_{j k}^{ \pm}(x, t ; y):=\frac{\left|x^{ \pm}\right|}{\left|a_{j}^{ \pm} t\right|} \beta_{j}^{ \pm}+\frac{|y|}{\left|a_{k}^{-} t\right|}\left(\frac{a_{j}^{ \pm}}{a_{k}^{-}}\right)^{2} \beta_{k}^{-}
$$

and

$$
\begin{aligned}
R(x, t ; y) & =\sum_{k} \mathbf{O}\left((t+1)^{-1 / 2} e^{-\eta x^{+}}+e^{-\eta|x|}\right) t^{-1 / 2} e^{-\left(x-y-a_{k}^{-} t\right)^{2} / M t} \\
& +\sum_{a_{k}^{-}>0, a_{j}^{-}<0} \chi_{\left\{\left|a_{k}^{-} t\right| \geq|y|\right\}} \mathbf{O}\left((t+1)^{-1 / 2} t^{-1 / 2} e^{-\left(x-a_{j}^{-}\left(t-\left|y / a_{k}^{-}\right|\right)\right)^{2} / M t} e^{-\eta x^{+}}\right. \\
& +\sum_{a_{k}^{-}>0, a_{j}^{+}>0} \chi_{\left\{\left|a_{k}^{-} t\right| \geq|y|\right\}} \mathbf{O}\left((t+1)^{-1 / 2} t^{-1 / 2} e^{-\left(x-a_{j}^{+}\left(t-\left|y / a_{k}^{-}\right|\right)\right)^{2} / M t} e^{-\eta x^{-}}\right.
\end{aligned}
$$

for some $\eta, M>0$, where $x^{ \pm}$denotes the positive/negative part of $x$, indicator function $\chi_{\left\{\left|a_{k}^{-} t\right| \geq|y|\right\}}$ is one for $\left|a_{k}^{-} t\right| \geq|y|$ and zero otherwise, indicator function $\chi_{\{t \geq 1\}}$ is one for $t \geq 1$ and zero otherwise, and scattering coefficients $\left[c_{k,-}^{0}\right],\left[c_{k,-}^{j, \pm}\right]$ are constant, with

$$
\sum_{a_{j}^{-}<0}\left[c_{k,-}^{j,-}\right] r_{j}^{-}+\sum_{a_{j}^{+}>0}\left[c_{k,-}^{j,+}\right] r_{j}^{+}+\left[c_{k,-}^{0}\right]\left(u_{+}-u_{-}\right)=r_{k}^{-}
$$

for each $k$ (note: uniquely determined, by condition (D2)), and

$$
\begin{aligned}
\sum_{a_{k}^{-}>0}\left[c_{k,-}^{0}\right] l_{k}^{-} & =\sum_{a_{k}^{+}<0}\left[c_{k,+}^{0}\right] l_{k}^{+} \\
& =\pi:=\left(r_{1}^{-}, \ldots, r_{p-1}^{-}, r_{p+1}^{+}, \ldots, r_{n}^{+}, u_{+}-u_{-}\right)^{-1} e_{n},
\end{aligned}
$$

where $e_{n}$ denotes the $n$th standard basis element. Likewise, we have the derivative 
bounds

$$
\begin{aligned}
\left|R_{x}\right| & =\sum_{k} \mathbf{O}\left((t+1)^{-1 / 2} t^{-1 / 2} e^{-\eta x^{+}}+e^{-\eta|x|}\right) t^{-1 / 2} e^{-\left(x-y-a_{k}^{-} t\right)^{2} / M t} \\
& +\sum_{a_{k}^{-}>0, a_{j}^{-}<0} \chi_{\left\{\left|a_{k}^{-} t\right| \geq|y|\right\}} \mathbf{O}\left((t+1)^{-1} t^{-1 / 2} e^{-\left(x-a_{j}^{-}\left(t-\left|y / a_{k}^{-}\right|\right)\right)^{2} / M t} e^{-\eta x^{+}}\right. \\
& +\sum_{a_{k}^{-}>0, a_{j}^{+}>0} \chi_{\left\{\left|a_{k}^{-} t\right| \geq|y|\right\}} \mathbf{O}\left((t+1)^{-1} t^{-1 / 2} e^{-\left(x-a_{j}^{+}\left(t-\left|y / a_{k}^{-}\right|\right)\right)^{2} / M t} e^{-\eta x^{-}}\right. \\
\left|R_{y}\right| & =\sum_{k} \mathbf{O}\left((t+1)^{-1 / 2} e^{-\eta x^{+}}+e^{-\eta|x|}\right) t^{-1} e^{-\left(x-y-a_{k}^{-} t\right)^{2} / M t} \\
& +\sum_{a_{k}^{-}>0, a_{j}^{-}<0} \chi_{\left\{\left|a_{k}^{-} t\right| \geq|y|\right\}} \mathbf{O}\left((t+1)^{-1} t^{-1 / 2} e^{-\left(x-a_{j}^{-}\left(t-\left|y / a_{k}^{-}\right|\right)\right)^{2} / M t} e^{-\eta x^{+}}\right. \\
& +\sum_{a_{k}^{-}>0, a_{j}^{+}>0} \chi_{\left\{\left|a_{k}^{-} t\right| \geq|y|\right\}} \mathbf{O}\left((t+1)^{-1} t^{-1 / 2} e^{-\left(x-a_{j}^{+}\left(t-\left|y / a_{k}^{-}\right|\right)\right)^{2} / M t} e^{-\eta x^{-}}\right.
\end{aligned}
$$

A symmetric decomposition holds for $y \geq 0$.

REMARKs. The stability criterion $(\mathcal{D})$ is equivalent to the Evans function condition of $[\mathrm{ZH}]$ (see Lemma 9.3 and Proposition 10.3, $[\mathrm{ZH}]$ ). The first condition is an obvious necessary condition for parabolic stability, while the second can be recognized (see, e.g. [M]) as the criterion for hyperbolic (i.e. inviscid) stability of the corresponding ideal shock; for further discussion, including the generalization to multidimensions, we refer the reader to [ZS] or [Z.4]. The bounds in Proposition 3.2 refine bounds obtained in $[\mathrm{ZH}]$ by similar methods. Notice that (3.11)-(3.15) reduce in the limiting case $\bar{u}(x) \equiv$ constant to the bounds obtained by Liu and Zeng [LZe] for stability of constant solutions.

We point out that condition (D1) is always satisfied in the scalar case (see e.g. discussions in [S,H,HZ.3,Z.3-4]), and, by results of Goodman, is satisfied in the system case for weak shocks of dissipative systems, satisfying the additional condition $L B R>$ 0 (see [G.1-2] and discussions in [ZH,Z.3-4]). Likewise, condition (D2) is satisfied always in the scalar case, for which $\Delta=u_{+}-u_{-}$, and, for weak shocks of systems, for which $r_{j}^{+} \sim r_{j}^{-}, r_{p}^{ \pm} \sim u_{+}-u_{-}$by Lax' (hyperbolic) shock structure theorem [La].

Interpretation/Discussion of Green's function bounds. From relation (3.17), the time asymptotic contribution of all excited terms is, similarly as in the scalar case, $\bar{u}_{x}\left\langle\pi, v_{0}\right\rangle$, where $\langle\cdot, \cdot\rangle$ denotes $L^{2}$ inner product, i.e. $\pi$ plays the role of a "left eigenfunction" at $\lambda=0$ dual to right eigenfunction $\bar{u}_{x}$; indeed, it is the effective left eigenfunction in the extended spectral theory of $[\mathrm{ZH}]$. Note, in the scalar case, that (3.17) reduces to the simple formula $\pi=\left(u_{+}-u_{-}\right)^{-1}$ given in Section 2 .

The form of vector $\pi$ can be deduced from first principles via $\pi \cdot\left(u_{+}-u_{-}\right)=1$ and the properties $\pi \cdot r_{k}^{-}=0, k=1, \ldots, p-1$, and $\pi \cdot r_{k}^{+}=0, k=p+1, \ldots, n$. These follow, in turn, from the observation that in the far fields "outgoing mass" in modes $r_{j}^{ \pm}, a_{j} \gtrless 0$, simply escapes to infinity, and cannot contribute to the shifting of the shock. This argument, and a heuristic treatment of scattering, first appeared in 
[LZ.2]; for related discussion, see [ZPM].

Along with excitation of the stationary mode $\bar{u}_{x}$, already seen in the scalar case, we have the new, system effect of scattering in the outgoing modes $r_{j}^{ \pm}, a_{j}^{ \pm} \gtrless 0$. Grouping together terms in $E, S$ with like initial propagation speeds $a_{k}^{-}$, we see that unit incoming mass in mode $r_{k}^{-}$, upon reaching the shock layer, splits into a portion $\left[c_{k,-}^{0}\right]$ accumulating at the shock and $n-1$ portions $\left[c_{k,-}^{j, \pm}\right]$ leaving the shock in the outgoing modes $r_{j}^{ \pm}, a_{j}^{ \pm} \gtrless 0$. More precisely, an initial delta-function perturbation at $y$ propagates in mode $r_{k}^{-}$as a Gaussian signal centered about $z_{k}^{-}:=y+a_{k}^{-} t$ until the time $T:=|y| /\left|a_{k}^{-}\right|$when it reaches the shock location $(z=0)$, thereafter splitting into a stationary wave centered around the shock and $n-1$ Gaussian signals outgoing in modes $r_{j}^{ \pm}$, centered about paths $z_{j k}^{ \pm}:=a_{j}^{ \pm}(t-T)$. The relation (3.16) thus represents conservation of mass for a single scattered signal; for further details, see [ZH,Z.1].

The form of the time-varying diffusion $\beta_{j k}^{ \pm}$in (3.14) may be easily understood in terms of the history of the scattered signal. For, evaluating to lowest order at the center $x=z_{j k}^{ \pm}$, in the critical regime $t \geq\left|y / a_{k}^{-}\right|$for which $z_{j k}^{ \pm} \gtrless 0$, we obtain the convex average

$$
\frac{\left|z_{j k}^{ \pm}\right|}{\left|a_{j}^{ \pm} t\right|} \beta_{j}^{ \pm}+\frac{|y|}{\left|a_{k}^{-} t\right|}\left(\frac{a_{j}^{ \pm}}{a_{k}^{-}}\right)^{2} \beta_{k}^{-},
$$

of the incoming diffusion $\beta_{k}^{-}$and a modified version

$$
\left(\frac{a_{j}^{ \pm}}{a_{k}^{-}}\right)^{2} \beta_{k}^{-}
$$

of the outgoing diffusion $\beta_{j}^{ \pm}$, weighted by the amounts of time $T=\left|y / a_{k}^{-}\right|$and $t-$ $T=\left|z / a_{j}^{ \pm}\right|$spent by the (center of the) signal in the respective modes $r_{k}^{-}$and $r_{j}^{ \pm}$. Correction (3.20) likewise has a simple geometric interpretation: For definiteness, consider a signal $z_{j k}^{+}$outgoing toward the positive side. During the time $T$ that the signal takes to reach the shock location $z=0$, it diffuses a distance $\sim\left(\beta_{k}^{-} T\right)^{1 / 2}$. This means that the trailing edge of the signal will strike the shock layer after the trailing edge by a difference of time $\Delta T \sim\left(\beta_{k}^{-} T\right)^{1 / 2} /\left|a_{k}^{-}\right|$, during which the two edges undergo convection differing by rate $a_{j}^{+}-a_{k}^{-}$. This results in additional, convective spreading of approximately

$$
\left(a_{j}^{+}-a_{k}^{-}\right) \Delta T \sim\left(\beta_{k}^{-} T\right)^{1 / 2}\left(a_{j}^{+}-a_{k}^{-}\right) /\left|a_{k}^{-}\right|,
$$

yielding a total distance of $\left(a_{j}^{+} / a_{k}^{-}\right)\left(\beta_{k}^{-} T\right)^{1 / 2}$, consistent with the corrected diffusion (3.20).

We point out two crucial differences between the bounds cited here and those reported earlier in $[\mathrm{ZH}]$. The first is the refined description (3.11) of the excited terms, replacing the cruder estimate

$$
E(x, t ; y)=\sum_{a_{k}^{-}>0}\left[c_{k,-}^{0}\right] \bar{u}_{x}(x) l_{k}^{-t} \chi_{\left\{\left|a_{k}^{-} t\right| \geq|x-y|\right\}}
$$


of $[\mathrm{ZH}]$; as we have seen already in the scalar case, this distinction is important for accurate wave-tracking incorporating diffusive effects (the dominant decay mechanism for nonlocalized data).

The second is the absence in $S_{y}, R_{y}$ of terms of the form

$$
\mathcal{O}\left(e^{-\eta|y|}\right) t^{-\frac{1}{2}} e^{-\frac{\left(x-y-a_{j}^{ \pm} t\right)}{M t}}
$$

$a_{j}^{ \pm} \gtrless 0$, corresponding to outgoing diffusion waves. Such terms necessarily occur for undercompressive shocks, hence must appear in the bounds of $[\mathrm{ZH}]$, which apply to shocks of all types.

EXAMPLE. In [LZ.1], the Green's function about an undercompressive stationary Complex Burger shock is obtained explicitly as

$$
G=\left(\begin{array}{cc}
G_{1} & 0 \\
0 & G_{2}
\end{array}\right)
$$

where $G_{1}$ is the Green's function (2.13) for a Real Burger shock, and

$$
G_{2}(x, t ; y):=\left(\frac{e^{\frac{y}{2}}}{e^{\frac{y}{2}}+e^{-\frac{y}{2}}}\right)(4 \pi t)^{-\frac{1}{2}} e^{-\frac{(x-y-t)^{2}}{4 t}}+\left(-\frac{e^{\frac{y}{2}}}{e^{\frac{y}{2}}+e^{-\frac{y}{2}}}\right)(4 \pi t)^{-\frac{1}{2}} e^{-\frac{(x-y+t)^{2}}{4 t}}
$$

We thus see directly that $\left(G_{2}\right)_{y}$ contains the terms

$$
\left(\frac{1}{2\left(e^{\frac{y}{2}}+e^{\left.-\frac{y}{2}\right)^{2}}\right.}\right)\left(-(4 \pi t)^{-\frac{1}{2}} e^{-\frac{(x-y-t)^{2}}{4 t}}+(4 \pi t)^{-\frac{1}{2}} e^{-\frac{(x-y+t)^{2}}{4 t}}\right) .
$$

As noted in [Z.1], on the other hand, terms of form (3.22) do not arise for Lax or overcompressive shocks. This is a vital observation, since the argument of the key Corollary 2.3 requires $\left|\tilde{G}_{y}\right|_{L^{p}(x)} \sim t^{-\frac{1}{2}(1-1 / p)-\frac{1}{2}}$, whereas

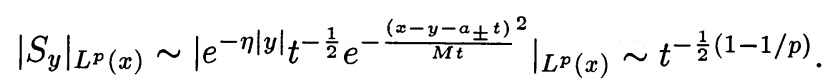

We therefore take a moment to explain this essential distinction in behavior:

A fundamental difference, pointed out with varying degree of rigor in [ZPM, LZ.2,ZH], between shocks of Lax/overcompressive type and shocks of undercompressive type is that scattering data - shock shift plus masses of outgoing diffusion waves - is in the former case entirely determined by mass of initial perturbation, but in the latter case depends also on the location (distribution) of that mass. eral form

Thus, scattering terms (comprising $S$ ) in the Lax/overcompressive case take gen-

$$
M_{j} t^{-\frac{1}{2}} e^{-\frac{\left(x-y-a_{j}^{ \pm} t\right)^{2}}{4 \beta_{j}^{ \pm} t}}
$$

where the coefficient matrices $M_{j} \in \mathbb{R}^{n \times n}$ are constant, and in the undercompressive case

$$
M_{j}(y) t^{-\frac{1}{2}} e^{-\frac{\left(x-y-a_{j}^{ \pm} t\right)^{2}}{4 \beta_{j}^{ \pm} t}}
$$


where $M_{j}(y)$ are asymptotically constant as $y \rightarrow \pm \infty$, but not constant. Differentiating (3.28) with respect to $y$, we see directly that $S_{y}$ in the undercompressive case contains additional terms

$$
M_{j}^{\prime}(y) t^{-\frac{1}{2}} e^{-\frac{\left(x-y-a_{j}^{ \pm} t\right)^{2}}{4 \beta_{j}^{\ddagger} t}} \sim e^{-\eta(y)} t^{-\frac{1}{2}} e^{-\frac{\left(x-y-a_{j}^{\ddagger} t\right)}{M t}}
$$

not present in the Lax/overcompressive case. The "Residual terms" $R_{y}$ are in both cases (Lax and undercompressive) faster decaying than $S_{y}$.

The rigorous proof of the above statements follows very much along the lines of our heuristic discussion. Precisely, to obtain the additional scattering information given in [Z.1], one has only to replace Proposition 7.1 in [ZH] by the more detailed Lemmas $4.7,4.21$, and 4.37 of [Z.4], which quantify these formal observations at the level of the resolvent kernel of the linearized operator about the wave. Then, approximating slowdecaying modes in $x$ by their constant-coefficient limits using Proposition 3.1 of [ZH] (a version of the "Gap Lemma" of [GZ]), one can proceed by exactly the same analysis as in $[\mathrm{ZH}]$, Theorem 8.3 to obtain the desired Green's function bounds via inverse Laplace transform. We point out that the form of $\beta_{j k}^{ \pm}$may be deduced from the saddlepoint estimate $c t^{-1 / 2} e^{-\bar{\alpha}^{2} / a_{k}^{-} p t}$ given in [ZH] for the scattered wave (now exact), where $\bar{\alpha}$ and $p$ are as defined in equation (8.79), p. 836 (resp. (8.91), p. 840) of that reference: specifically, from $\bar{\alpha}=\left(a_{k}^{-} / a_{j}^{ \pm}\right)\left(x-z_{j k}^{ \pm}\right) / 2 t$, we obtain $\beta_{j k}^{ \pm}=p\left(a_{j}^{ \pm}\right)^{2} / a_{k}^{-}$, yielding the result. The refined description of the excited term may be obtained similarly, using Lemmas 4.7, 4.21, and 4.37 of [Z.4], together with Proposition 3.1 of [ZH] to obtain the principal part as an explicit Fourier integral. For details, see [Z.1].

REMARK 3.3. Further considerations suggest that improved $y$-derivative bounds do not hold in the system case for the "integrated equations"

$$
v_{t}=\mathcal{L} v:=-A v_{x}+B v_{x x},
$$

arising through the flux transform of [G.2,GY], nor do second-derivative bounds for the "unintegrated" equations exhibit further improvement; that is, the bounds available by the methods of [Z.1] are sharp. For, consider the trivial case of a Lax shock $\bar{u}(x)=(-\tanh (x / 2), 0)$ of $(3.1)$ with $f\left(u_{1}, u_{2}\right):=\left(u^{2} / 2,(u+2) v\right)^{t}$ and $B \equiv I$, for which equations (3.30) decouple into

$$
\left(v_{1}\right)_{t}=-a_{1}\left(v_{1}\right)_{x}+\left(v_{1}\right)_{x x}
$$

and

$$
\left(v_{2}\right)_{t}=-a_{2}\left(v_{2}\right)_{x}+\left(v_{2}\right)_{x x}, \quad a_{2} \geq 1>0,
$$

and the Greens function $\mathcal{G}$ into

$$
\mathcal{G}=\left(\begin{array}{cc}
\mathcal{G}_{1} & 0 \\
0 & \mathcal{G}_{2}
\end{array}\right)
$$

The first equation, corresponding to the principal characteristic field, is just that arising from a scalar shock of (integrated) Burgers equation), hence obeys the derivative bound $\left|\left(\mathcal{G}_{1}\right)_{y}\right|_{L^{1}(x)} \sim t^{-1 / 2}$ (by the bounds of [ZH], or direct computation similar to (2.13)). The second equation, corresponding to the transverse characteristic field, 
may be studied by the observation that $\left(\mathcal{G}_{2}\right)_{y}=G_{x}$, where $G$ is the Green's function for

$$
v_{t}=-\left(a_{2} v\right)_{x}+v_{x x}
$$

hence $\left(\mathcal{G}_{2}\right)_{y}=\int_{x} G_{y y}$. Heuristically, we expect

$$
G \sim K(x-z(y, t), t),
$$

where $K(x, t):=(4 \pi t)^{-1 / 2} e^{-x^{2} / 4 \pi t}$ denotes a standard heat kernel and $z(y, t)$ denotes the path of a signal originating at $y$ and convected at rate $a_{2}$, hence

$$
\left|z_{y}\right| \sim\left|a-a_{ \pm}\right| \sim e^{-\eta|y|}
$$

for large $t$. Thus, $G_{y} \sim-z_{y} K_{x} \sim e^{-\eta|y|} K_{x}$, matching the predictions above, but

$$
G_{y y} \sim-z_{y y} K_{x}-z_{y}^{2} K_{x x} \sim e^{-\eta|y|} K_{x}
$$

and

$$
\left(\mathcal{G}_{2}\right)_{y} \sim-z_{y y} K-z_{y}^{2} K_{x} \sim e^{-\eta|y|} K,
$$

whence $\left|G_{y y}\right|_{L^{1}(x)} \sim\left|G_{y}\right|_{L^{1}(x)} \sim t^{-1 / 2}$ and $\left|\left(\mathcal{G}_{2}\right)_{y}\right|_{L^{1}(x)} \sim|K|_{L^{1}(x)} \sim 1>>t^{-1 / 2}$.

To put it another way, more related to the previous discussion of the undercompressive case, (3.32) does not exhibit conservation of mass, hence we expect behavior

$$
\mathcal{G}_{2} \sim m(y, t) K(x-z(y, t))
$$

in place of (3.33), with $m_{y} \sim e^{-\eta|y|}$. This yields directly that $\left(\mathcal{G}_{2}\right)_{y} \sim m_{y} K \sim e^{-\eta|y|} K$, matching conclusion (3.34) above.

Nonlinear stability analysis. With these observations, our nonlinear stability analysis carries through exactly as in the scalar case. That is, defining $v, \delta$ formally by $(2.29),(2.35)$ and $\widetilde{E}, \widetilde{G}, \widetilde{e}$ by $(2.16)$ and $(2.19)$, we again arrive at the reduced equations (2.36)-(2.37). Likewise by essentially the same calculations as in the scalar case, we have:

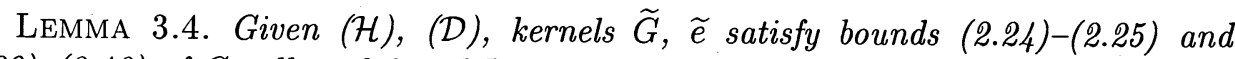
(2.39)-(2.40) of Corollary 2.3 and Lemma 2.5, respectively.

Proof. The crucial estimation of $E-\tilde{E}$ (resp. $E_{y}-\tilde{E}_{y}$ ) is obtained by summing over each incoming scalar mode $k, a_{k}^{-}>0$, the estimates from the scalar case, while the terms $S, R$ (resp. $S_{y}, R_{y}$ ) again clearly absorb.

Thus, the arguments of Proposition 2.4 and Theorem 2.7/Corollary 2.8 carry over verbatim to yield our main theorems:

THEOREM 3.5. Given $(\mathcal{H}),(\mathcal{D})$ is necessary and sufficient for $L^{p}$-linearized orbital stability of $\bar{u}(\cdot)$ with respect to initial perturbations $v_{0} \in L^{1}$ or $\left|v_{0}(x)\right| \leq C(1+$ $|x|)^{-r}, r>1 / p$. Moreover, if (D) holds, we have the (sharp) linear decay bounds:

$$
|v(\cdot, t)-\varphi(\cdot, t)|_{L^{p}} \leq t^{-\frac{1}{2}(1-1 / p)}\left|v_{0}\right|_{L^{1}},
$$

in the first case, and in the second case

$$
|v(\cdot, t)-\varphi(\cdot, t)|_{L^{p}} \leq t^{-\frac{1}{2}(1 / \tilde{p}-1 / p)}\left|v_{0}\right|_{L^{\tilde{p}}}
$$


for all $p \geq \tilde{p}>1 / r$, where $v$ is the solution of (3.6) with initial data $v_{0}$, and $\varphi$ is as defined in (2.15)- (2.19).

Theorem 3.6. Let (H), (D) hold. Then, for $\left|u_{0}-\bar{u}\right|_{L^{1}},\left|u_{0}-\bar{u}\right|_{L^{\infty}} \leq \zeta_{0}, \zeta_{0}$ sufficiently small, the solution $u(x, t)$ of (3.2) with initial data $u_{0}$ satisfies

$$
|u(x, t)-\bar{u}(x-\delta(t))|_{L^{p}} \leq C \delta_{0}(1+t)^{-\frac{1}{2}(1-1 / p)} .
$$

where $\delta(t)$ (defined as above) satisfies

$$
\begin{gathered}
\left(\delta(t) \mid \leq C \zeta_{0}(1+t)^{-\frac{1}{2}},\right. \\
|\delta(t)| \leq C \zeta_{0} .
\end{gathered}
$$

REMARKS. 1. The conclusions of Theorem 3.5 apply also for over- and undercompressive shocks (note: this result requires only bounds on $|G|$ and not $\left|G_{y}\right|$, which are equivalent in the three cases [Z.1]).

2. For localized data, $|v| \sim(1+|x|)^{-r}, r>1$, pointwise bounds analogous to those of Theorem 2.9 can also be obtained, but here there is not particular advantage of the Lax over the undercompressive case; for this analysis, we refer the reader to [Z.2].

3. As noted above, the overcompressive case features the same improved $y$ derivative Green's function bounds as does the Lax case; however, the stationary manifold $\left\{\bar{u}^{\delta}\right\}$ of solutions of (3.1)-(3.2) local to $\bar{u}$ does not typically have the simple group structure used in our stability argument (translation, in the Lax and undercompressive case). It is an interesting open question whether $L^{1} \cap L^{\infty} \rightarrow L^{p}$ stability holds in this case. Nonlinear stability of overcompressive shocks was shown in [ZH] using a pointwise argument of Liu $[\mathrm{L}]$, for data decaying as $(1+|x|)^{-3 / 2}$.

\section{REFERENCES}

[FS] H. FreistüHLER AND D. SERRE, $L^{1}$ stability of shock waves in scalar viscous conservation laws, Comm. Pure Appl. Math., 51:3 (1998), pp. 291-301.

[Fr] A. Friedman, Partial Differential Equations of Parabolic Type, Prentice-Hall, Englewood Cliffs, NY, 1964, Reprint Ed. (1983).

[G.1] J. Goodman, Nonlinear asymptotic stability of viscous shock profiles for conservation laws, Arch. Rat. Mech. Anal., 95 (1986), pp. 325-344.

[G.2] J. Goodman, Remarks on the stability of viscous shock waves, in Viscous profiles and numerical methods for shock waves (Raleigh, NC, 1990), SIAM, Philadelphia, PA, 1991, pp. $66-72$.

[GSZ] J. Goodman, A. Szepessy, ANd K. Zumbrun, A remark on the stability of viscous shock waves, SIAM J. Math Anal, 25:6 (1994), pp. 1463-1467.

[GY] J. Goodman AND A. YIP, Private communication.

[He] D. Henry, Geometric Theory of Semilinear Parabolic Equations, Lecture Notes in Mathematics, Springer-Verlag, Berlin, 1981 iv + 348 pp.

[H.1] Peter Howard, Pointwise estimates for the stability of a scalar conservation law, Thesis, Indiana University (1998).

[H.2] Peter Howard, Pointwise estimates on the Green's function for a scalar linear convectiondiffusion equation, J. Differential Equations, 155:2 (1999), pp. 327-367. 
[H.3] P. HowARd, Pointwise Green's function approach to stability for scalar conservation laws, Comm. Pure Appl. Math., 52:10 (1999), pp. 1295-1313.

[HZ.1] P. Howard AND K. Zumbrun, Pointwise estimates and stability for dispersive-diffusive shock waves, Preprint (1999).

[HZ.2] P. HowARD AND K. ZUMBRUn, Tracking of viscous shock waves, Preprint (1999).

[IO] IL'IN AND OLEINIK, Behavior of the solution of the Cauchy problem for certain quasilinear equations for unbounded increase of time, AMS Translations, 42 (1964), ser. 2, pp. 19-23.

[K] S. KAWASHIMA, Systems of a hyperbolic-parabolic composite type, with applications to the equations of magnetohydrodynamics, thesis, Kyoto University (1983).

[La] P. LAX, Hyperbolic systems of conservation laws II, Comm. Pure Appl. Math., 10 (1957), pp. 537-566.

[Le] E.E. LEVI, Sulle equazioni lineari totalmente ellittiche alle derivate parziali, Rend. Circ. Mat. Palermo, 24 (1907), pp. 275-317.

[L] T.-P. LIU, Nonlinear stability of shock waves for viscous conservation laws, Mem. Amer. Math. Soc., 56 (1985), no. 328, v+108 pp.

[LZe] T.-P. LiU And Y. ZENG, Large Time Behavior of Solutions for General Quasilinear Hyperbolic-Parabolic Systems of Conservation Laws, AMS memoirs 599, 1997.

[LZ.1] T.-P. LIU AND K. ZUMBRUN, Nonlinear stability of an undercompressive shock for complex Burgers equation, Commun. Math. Phys., 168 (1993), pp. 163-186.

[LZ.2] T.-P. LIU AND K. ZUMBRUN, On nonlinear stability of general undercompressive viscous shock waves, Commun. Math. Phys., 174 (1995), pp. 319-345.

[M] A. MAJDA, The stability of multidimensional shock fronts, Mem. Amer. Math. Soc., 41 (1983), no. 275 , pp. iv +95 pp.

[MP] A. MAJDA AND R. PEgO, Stable viscosity matrices for systems of conservation laws, J. Diff. Eqs., 56 (1985), pp. 229-262.

[N] K. NishinaRA, A note on the stability of travelling wave solutions of Burgers' equation, (English) Japan. J. Appl. Math., 2:1 (1985), pp. 27-35.

[S] D. SATtinger, On the stability of waves of nonlinear parabolic systems, Adv. Math., 22 (1976), pp. 312-355.

[SX] A. Szepessy AND Z. XIN, Nonlinear stability of viscous shock waves, Arch. Rat. Mech. Anal., 122 (1993), pp. 53-103.

[SZ] A. Szepessy AND K. Zumbrun, Stability of rarefaction waves in viscous media, Arch. Rational Mech. Anal., 133:3 (1996), pp. 249-298.

[Z.1] K. Zumbrun, Refined Green's function bounds for stability of viscous shock waves, in preparation (1999).

[Z.2] K. ZumbRun, Stability of general undercompressive shocks of viscous conservation laws, in preparation.

[Z.3] K. Zumbrun, Stability of viscous shock waves, Lecture Notes, Indiana University (1998).

[Z.4] K. Zumbrun, Multi-dimensional stability of viscous shock fronts, Lecture notes, TMR summer school, Köchel, May 1999.

[Z.5] K. Zumbrun, Formation of diffusion waves in a scalar conservation law with convection, Trans. Amer. Math. Soc., 347:3 (1995), pp. 1023-1032.

[ZH] K. Zumbrun AND P. HowARD, Pointwise semigroup methods and stability of viscous shock waves, Indiana Mathematics Journal, V47:4 (1998), pp. 741-871.

[ZS] K. ZUMBRUN AND D. SERRE, Viscous and inviscid stability of multi-dimensional planar shock fronts, to appear, Indiana Math. J. (1999).

[ZPM] K. Zumbrun, B. Plohr, AND D. MARChesin, Scattering behavior of transitional shock waves, Mat. Contemp., 3 (1992), pp. 191-209. 